\title{
Das Wachstum des Geweihes von Capreolus vulgaris.
}

\author{
Von \\ Forstmeister Dr. Adolf Rörig \\ in Frankfurt a. M. \\ Mit Tafel XVIII. \\ Eingegangen am 10. Dezember 1907.
}

Im Laufe des Jahres 1905 war es mir möglich gewesen, das Wachstum des Geweihes von Cervus elaphus, von C. barbarus und von C. canadensis fortgesetzt beobachten und vom morphologischbiologischen Standpunkte aus untersuchen zu können. Die hierbei gewonnenen Resultate wurden im 20. Bande dieses Archivs veröffentlicht.

Es lag nun nahe, auch das Wachstum des Rehgeweihes nach gleichem Verfahren einer genauen Beobachtung und Untersuchung zu unterziehen.

Ein hierzu geeignetes Individuum zur Verfügung zu bekommen, wollte jedoch nicht so bald gelingen. Nicht immer besitzen die in Gefangenschaft gehaltenen Rehböcke die zu genanntem Zwecke erforderlichen Eigenschaften und an in Freiheit lebenden Individuen können Beobachtungen der beabsichtigten Art selbstverständlich nicht vorgenommen werden.

Das Wachstum des Geweihes dieser Species eingehend zu beobachten, mußte nun aber gerade von besonderem Interesse sein und zwar aus dem Grunde, weil Capreolus in der Reihe der Cerviden eine vollkommen isolierte Stellung einnimmt. Eine Verwandtschaft von Capreolus mit den Cerviden der Alten Welt ist aus anatomischen Rücksichten gänzlich ausgeschlossen. Alle Cerviden des genannten Erdteiles besitzen plesiometacarpale Vorderextremitäten, während die des Rehes telemetacarpal gebaut sind. Dagegen besteht in dieser Hinsicht tatsächlich eine Verwandtschaft von Capreolus mit den Cerviden Amerikas. Von letzteren ist Capreolus jedoch wieder getrennt 
durch die Art des anatomischen Baues des Vomer, der jenem der altweltlichen Cerviden vollkommen gleicht. Die dem Vomer anfsitzende senkrechte knöcherne Platte des Riechrohres ist holorbin beim Reh wie bei den Cerviden der Alten Welt; sie ist tichorhin bei allen Cerviden Amerikas, sie teilt das Riechrohr dieser in zwei bis zu den Choanen reichende völlig gesonderte Abteilungen.

Was nun das Geweih von Capreolus betrifft, so ist zu bemerken, daß dasselbe den Geweihen amerikanischer Cerviden näher steht als denen der Alten Welt; nicht e in e Species der letzteren besitzt einen dem Rehgeweih auch nur entfernt ähnlichen Bau. Der Unterschied zwischen diesen besteht hauptsächlich in der Anwesenheit einer rückwärts gerichteten Sprosse an jeder Stange des Rehgaweihes, die in dieser Form an keinem Geweih altweltlicher Hirsche sich findet. Dagegen besitzen die Geweibe aller amerikanischen Hirsche, welche die Gabelstufe tiberholen, eine Sprosse dieser Art.

Ferner ist das Rehgeweih im Gegensatz zu den Geweihen der altweltlichen Hirsche durch den Mangel einer wirklichen Augsprosse ausgezeichnet, ein Mangel, durch welchen auch die Geweihe der autochthonen amerikanischen Hirsche, mit Ausschluß von Rangifer, charakterisiert sind. Die Geweihbildung von Capreolus zeigt also größere Verwandtschaft mit derjenigen amerikanischer Cerviden als mit derjenigen altweltlicher Hirsche.

Auch hinsichtlich der Jahreszeit, in welcher der Geweihwecbsel stattfindet, besteht Übereinstimmung zwischen dem Reh und den nordamerikanischen Cerviden; dieser Wechsel vollzieht sich bei den Angehörigen dieser Gruppe während der Wintermonate, bei den Cerviden der Alten Welt im Frïhjahr und Sommer.

Das Reh nimmt also zwischen alt- und neuweltlichen Cerviden eine Mittelstellung ein, steht aber in Hinsicht der Telemetacarpalie, der Geweihbildung und des Geweihwechsels den letzteren näher als den altweltlichen Cerviden, mit denen es nur die Holorhinie gemein hat.

Hiernach ergibt sich, daß es von Interesse sein mußte, das Wachstum gerade des Rehgeweihes eingehend zu studieren.

Glïcklicherweise fand sich denn auch nach längerem Snchen ein hierzu vorzüglich geeignetes Individuum. Dasselbe zeigte in jeder Hinsicht alle Eigenschaften, die erwarten ließen, daß der Aufbau des neuen Geweihes in typischer und normaler Weise sich vollziehen würde. Der Raum, der diesem Exemplar zur Verfugung stand, besaß Dimensionen von solcher Lünge und Breite, daß er dem Gefangenen genügende Bewegung gewährte. Er bot ihm auch, da er von 
Laub- und Nadelhölzern licht bestanden war, nach Bedürfnis Sonnenwärme und Schatten. $\mathrm{DaB}$ der Rehbock sich hier wohlbefand, bekundete er durch lebhafte Sprünge, wenn er sich zuweilen zu solchen veranlaßt fühlte. Die Konstitution des Körpers konnte als eine gute bezeichnet werden. Sein Alter betrug zu Beginn meiner Beobachtungen 2 Jahre 6 Monate.

Vor allem bot das Geweih, welches der Rehbock noch um die Mitte des November trug, Garantie sowobl für das Wohlbefinden dieses Exemplars als auch für die Wahrscheinlichkeit der Entwicklung einer typischen Neubildung. Nachdem dieses Geweih abgeworfen und montiert worden war, habe ich eine genaue Zeichnung bzw. Abbildung davon entworfen, welche hier unter Fig. 1 reproduziert worden ist und dazu dienen soll, das vorstehend Erwähnte zu bekräftigen, sowie einen Vergleich zwischen ihm und dem neu entwickelten Geweih zu ermöglichen.

Bevor ich den Hergang meiner Untersuchungen hier schildere, gestatte ich mir einige Bemerkungen in betreff der Entwicklungsgeschichte des Objekts meiner Studien.

Der betreffende Rehbock war im Mai 1904 zur Welt gekommen. Vom Dezember 1904 bis zum März 1905 produzierte er das von mir so genannte »Primitivgeweih«. Dieses warf er im März 1905 ab und entwickelte nun das erste Spießgeweih, das er bis zum Dezember des genannten Jahres trug. Nach Abwurf dieses Spießgeweihes, das eine Länge von $7 \mathrm{~cm}$ erreicht hatte, produzierte dieser Rehbock ein Geweih, das unter Fig. 1 in halber natürlicher Größe abgebildet worden ist. Die Dimensionen dieses Geweihes sind nun folgende: Umfang jeder der beiden Rosen 12,5 cm, Höhe der Stangen $17 \mathrm{~cm}$, Durchmesser derselben oberhalb der Rosen $2,3 \mathrm{~cm}$, Dimension vom Rosenstock bis zur Gabelbucht der Vordersprosse rechts 10,5, links $11 \mathrm{~cm}$, Länge dieser Sprosse 1,7 cm, Dimension vom Rosenstock bis zur Gabelbucht der Hintersprosse 13,5 cm, Länge dieser Sprosse 3,8 cm, Länge des Stangenendes $4,5 \mathrm{~cm}$.

Der Abwurf der rechtsseitigen Stange dieses Geweihes geschah am 25. November, derjenige der linken Stange am 27. November 1906.

Die das Geweihwachstum betreffenden Beobachtungen nahmen am 26. November ihren Anfang. Es wurde bei Ausführung dieser Beobachtungen und Untersuchungen genau dieselbe Methode befolgt, wie solche gelegentlich der Feststellung des Wachstums des Geweihes der oben genannten drei Edelhirscharten zur Anwendung gekommen 
war, und die ich in meinem diesen Gegenstand behandelnden Artikel ausführlich beschrieben habe.

Am 26. November, also an dem Tage nach dem Abwurf der rechtsseitigen Geweihstange, war die Abwurffläche auf dem Stirnzapfen mit dunkelvioletter Haut überzogen, die Mitte der Fläche nahm ein runder, $12 \mathrm{~mm}$ im Querdurchmesser haltender Schorf ein.

Nach Ablauf der ersten, 14 Tage umfassenden Wachstumsperiode, am 10. Dezember, hatte die Neubildung auf jedem der beiden Stirnzapfen einen Querdurchmesser von 2,6 cm, nach Abzug des die Neubildung bedeckenden Integuments einen solchen von $2,0 \mathrm{~cm}$ und eine Höhe von $1 \mathrm{~cm}$. Der kubische Inhalt betrug also für jede der beiden Neubildungen 3,14 Kubikzentimeter (ccm). Der Schorf auf der oberen Fläche der Neubildung hatte nur noch $4 \mathrm{~mm}$ Durchmesser. Das Gebilde war fast gänzlich unbehaart. Der mit Ziffer 1 bezeichnete Geweihteil der Fig. 2 stellt die in dieser Periode an einer Geweihstange produzierte Neubildung dar.

Die Geringftugigkeit dieser Neubildungsgröße erklärt sich daraus, daß im Laufe dieser Wachstumsperiode der Stirnzapfen zunächst diejenige Stärke erlangen mußte, welche der Stärke des neu aufzubauenden Geweihes entsprach.

In der zweiten Wachstumsperiode vom 10. bis 24. Dezember, also in der dritten und vierten Woche, hatte die Neubildung einen Querdurchmesser von $3,0 \mathrm{~cm}$ und eine Höhe von 4,5 cm erlangt, wovon auf diese Wachstumsperiode $3,5 \mathrm{~cm}$ entfielen. Die Stärke des Integuments zu $3 \mathrm{~mm}$ gerechnet, bleibt für das eigentliche Geweih 2,4 cm Querdurchmesser. Der kubische Inhalt der Geweihstange betrug nun 15,85 ccm. Der Schorf war in einer Höhe von $4 \mathrm{~cm}$ an der Innenseite der Neubildung verlagert. Diese exzentrische Verlagerung des Schorfes war die Folge der allmählichen Verschiebung der Stangenachse nach der Außenseite. Feine kurze Härchen bedeckten nun die Neubildung. Der mit Ziffer 2 bezeichnete Teil der Fig. 2 stellt die zweite Neubildung dar.

In der dritten Wachstumsperiode vom 24. Dezember 1906 bis 7. Januar 1907, also in der fünften und sechsten Woche, hatte die gesamte Neubildung eine Höhe von etwa $10 \mathrm{~cm}$ erreicht, davon entfielen auf die Neubildung dieser Periode $5,5 \mathrm{~cm}$. Der Querdurchmesser derselben betrug einschließlich des Integuments $3 \mathrm{~cm}$, ohne dasselbe $2,4 \mathrm{~cm}$. Der kubische Inhalt berechnet sich hiernach auf 24,91 ccm. Der mit Ziffer 3 bezeichnete Teil der Fig. 2 bringt die dritte Neubildung zur Darstellung. 
Bemerkenswert ist, daß sich bei Ablanf dieser Wachstumsperiode die ersten Spuren einer Gabelung am oberen Ende der Neubildung zeigten, welche auf die Entstehung der Vordersprosse hinweist. Hier betrug die Dimension in der Richtung von vorn nach hinten $3,5 \mathrm{~cm}$. Am unteren Ende der gesamten Neubildung machten sich die Anfänge der Rosenbildung bemerkbar. Die Entwicklang einer Rose beginnt also auch am Geweih des Rehbocks keineswegs gleich bei Beginn der Entstehung einer Geweihneubildung - wie oft noch irrigerweise angenommen wird - sondern stets erst gegen die Mitte des gesamten Wachstumszeitraumes.

In der vierten Wachstumsperiode vom 7 . bis 21. Januar, also in der siebenten und achten Woche, hatte die Geweihstange eine Höhe von $17 \mathrm{~cm}$ erreicht, wovon auf die Neubildung dieser vierten Periode $7 \mathrm{~cm}$ entfallen. Überdies war nun der größte Teil der Vordersprosse und ein Teil der Hintersprosse entwickelt worden. Die Länge der Vordersprosse (nebst einem Teil der Geweihstange) betrug $3,6 \mathrm{~cm}$, diejenige der Hintersprosse $\mathrm{maB} 3,2 \mathrm{~cm}$. Der Querdurchmesser der Geweihstange betrug einschließlich des Integuments 2,3, ohne dasselbe $1,7 \mathrm{~cm}$, der Querdurchmesser der Vordersprosse maß einschließlich des Integuments 1,6, ansschließlich desselben $1,0 \mathrm{~cm}$, der der Hintersprosse betrug einschließlich des Integuments 2,2, ohne dasselbe $1,6 \mathrm{~cm}$. Hiernach berechnet sich der kubische Inhalt insgesamt auf $25,18 \mathrm{ccm}$. Ziffer 4 bezeichnet bei Fig. 2 den in dieser Periode durch Wachstum erworbenen Abschnitt der gesamten Neubildung. Die Entwicklung der Rose hatte weitere Fortschritte gemacht. Die Kolben des Geweihes waren nun dicht mit Haaren bedeckt.

In der funften Wachstumsperiode vom 21. Januar bis 4. Februar, also in der neunten und zehnten Woche, hatte die Geweihstange eine Höhe von $21 \mathrm{~cm}$ erlangt. Hiervon entfielen auf diese fünfte Periode $4 \mathrm{~cm}$. An der Vordersprosse hatte ein Zuwachs von $1 \mathrm{~cm}$, an der Hintersprosse ein solcher von 2,6 cm stattgefunden. Der Querdurchmesser der Neubildung betrug an der Geweihstange mit dem Integument $1,6 \mathrm{~cm}$, ohne dasselbe $1,2 \mathrm{~cm}$, an der Vordersprosse betrug er mit dem Integument 0,6 , ohne dasselbe $0,2 \mathrm{~cm}$, an der Hintersprosse mit dem Integument 0,9 , obne dasselbe $0,5 \mathrm{~cm}$. Der kubische Inhalt berechnet sich hiernach insgesamt auf $5,06 \mathrm{ccm}$. Die mit Ziffer 5 bezeichneten Teile der Fig. 2 stellen die Neubildnng dieser Periode dar. Die Entwicklung der Rose hatte nun starke Fortschritte gemacht. 
In der sechsten Wachstumsperiode vom 4. bis 18. Februar, also in der elften und zwölften Woche, gelangte das äuBerlich erkennbare Wachstum des Geweihes zum AbschluB. Der Zuwachs in die Länge betrug an der Geweihstange nur noch $1,5 \mathrm{~cm}$, an der Hintersprosse $0,6 \mathrm{~cm}$. Der Querdurchmesser der Neubildung betrug an der Stange mit dem Integument 0,8 , ohne dasselbe $0,4 \mathrm{~cm}$, an der Hintersprosse mit dem Integument 0,5 , ohne dasselbe $0,2 \mathrm{~cm}$. Hiernach berechnet sich der kubische Inhalt der letzten Neubildung insgesamt auf 0,21 ccm. Ziffer 6 bezeichnet an Fig. 2 den in dieser Periode produzierten Geweihzuwachs.

Das äuBerlich erkennbare Geweihwachstum war hiermit also beendet. Zum Aufbau des Geweihes waren demnach zwölf Wochen oder 84 Tage erforderlich gewesen. Das Geweih hatte eine Höhe von 22,5 cm erreicht; die Lüngendimension der Vordersprosse betrug (abzüglich des bei der kubischen Inhaltsberechnung vom Stangenteil hinzugezogenen Abschnitts) 4,0 cm, die der Hintersprosse etwa $6,0 \mathrm{~cm}$. Der durch Wachstum erworbene kubische Inhalt einer Geweihstange betrug 74,35 ccm, und der des ganzen Geweihes $148,70 \mathrm{ccm}$.

Wie an Fig. 2 ersichtlich, war der Gang des Wachstums ein ungleichmäßiger, d. h. innerhalb gleich langer Zeitperioden wurden ungleich große Zuwachsbeträge erzielt. Es betrug die Zuwachsgröße für eine Geweihhälfte in der

\begin{tabular}{|c|c|c|c|c|c|c|}
\hline & msperiode & . & 3,14 & $\mathrm{~cm}$ & $=$ & $4,2 \%$ \\
\hline 2. & - & . & 15,85 & - & $=$ & $21,3-$ \\
\hline 3. & - & 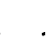 & 24,91 & - & $=$ & 33,5 \\
\hline 4. & - & . & 25,18 & - & $=$ & 33,9 \\
\hline 5. & - & . & 5,06 & - & $=$ & 6,8 \\
\hline 6. & - & . & 0,21 & - & $=$ & 0,3 \\
\hline
\end{tabular}

Der stärkste Zuwachs am Geweih hatte demnach innerbalb der fünften bis achten Woche stattgefunden. Innerhalb dieses etwa 4 Wochen betragenden und die Mitte der ganzen Wachstumszeit einnehmenden Zeitraumes waren also zwei Drittel der gesamten Zuwachsgröße durch Wachstum am Geweih produziert worden, während innerhalb der 8 Wochen umfassenden, am Anfang und am Ende der Wachstumszeit gelegenen Zeiträume nur ein Drittel der totalen Zuwachsgröße zustande gekommen war.

Die in den einzelnen Wachstumsperioden erfolgten Längezunahmegrößen betrugen in der 
1. 2. 3. $\quad$ 4. 5. $\quad$ 6. Wachstumsperiode an der Geweihstange $\quad 1,0 \quad 3,5 \quad 5,5 \quad 7,0 \quad 4,0 \quad 1,5=22,5 \quad \mathrm{~cm}$

- - Vordersprosse - - - 3,0 1,0 - = 4,0 -

- Hintersprosse - - $-3,2$ 2,6 $0,6=6,0$ -

Die Entwicklnng der Rose begann gegen das Ende der vierten Wachstumsperiode, in der achten Woche; sie erreichte ihren Abschluß gegen das Ende der sechsten Periode, in der zwölften Woche.

Nit der Beendigung des äußerlich erkennbaren Wachstums behielt das Integument noch seine ursprüngliche straffe Spannung, weil in den inneren Teilen des Geweihes der Verknöcherungsprozeß noch seinen Fortgang nahm. Selbst noch am 18. März, also 4 Wochen nach Beendigung des äußeren Geweihaufbanes, war eine Schrumpfung des Integuments nicht festzustellen, ein Beweis also, daß die Verknöcherung im Innern des Geweihes noch nicht abgeschlossen war.

Nachdem nun auch das Wachstum im Innern des Geweihes sein Ende erreicht hatte, das Geweih also vollkommen ausgereift war, begann das Integument zu schrumpfen und trat der Zeitpunkt des Fegens ein. Das Fegen begann am 30. März und zog sich etliche Tage hin. Verzögerungen dieser Art sind bei in Gefangensehaft gehaltenen Individuen keine Seltenheit; Rehböcke in der Freiheit werden mit diesem Geschäft gewöhnlich in einer Nacht fertig.

Der Aufbau und das Ausreifen des Geweihes von diesem Rehbock hat demnach 18 Wochen in Anspruch genommen.

Bemerkt sei noch, daß die Verfärbung dieses Individuums bereits Mitte Februar ihren Anfang nabm, und zwar gleichzeitig an den unteren Teilen der vier Extremitäten.

Einen Vergleich der Wachstumsresultate, die am vor- und diesjährigen Geweih des hier bebandelten Rehbockes erzielt worden sind, liefert folgende Zusammenstellung:

Höhe des ganzen Geweihes . .
Dimension vom Stirnzapfen bis zur

Gabelbucht der Vordersprosse. . 10,5-11 - 11,5 desgleichen bis zur Gabelbucht der

Hintersprosse . .

Länge der Vordersprosse . . . .

- - Hintersprosse . . . . 3,8 -

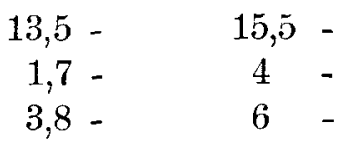

Die durch einjährigen Zuwachs erzielten Größen der Geweihdimensionen ergeben sich hier durch Vergleich von selbst. 
Die Frage, ob sich in dem Modus, des Geweihwachstums, d. h. in dem Geweihwachstumsgange, bei einem Vergleiche zwischen Rehbock und Edelhirsch ein bemerkenswerter Unterschied herausgestellt hat, läßt sich absolut weder bejahen noch verneinen. Dies liegt an der Ungleichheit der Zahl der an Zeitdauer gleich großen Wachstumsperioden, die beim Edelhirsch neun, beim Rehbock nur sechs beträgt. Dies geht aus der hier folgenden Gegenuberstellung der den kubischen Inhalt bezeichnenden $\mathrm{Zu}$ wachsprozente in den einzelnen Wachstumsperioden hervor, die trotzdem nicht des Interesses entbehrt.

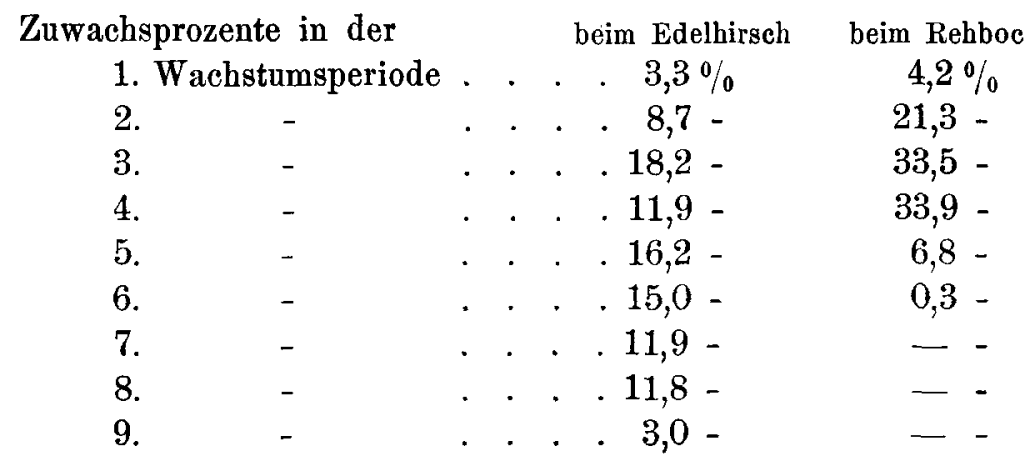

Wie ersichtlich, steigt die Geweihzuwachsgröße beim Rehbock schon mit Beginn der zweiten Wachstumsperiode rapid an, um in der dritten Periode die höchste Wachstumsenergie zu erreichen und dieselbe in der folgenden Periode auch zu behaupten; und nun sinkt diese Energie fast ebenso rapid, um dann in der sechsten und letzteu Periode zu einem schnellen $A b s c h l n B$ zu gelangen.

Abweichend hiervon ist der Wachstumsgang des Geweihaufbaues vom Edelhirsch. In den ersten beiden Perioden ist das Ansteigen der Zuwachsgröße mäßig; diese erreicht aber bereits in der dritten Periode ihr höchstes $\mathrm{Maß}$; sie erhält sich bei geringen Schwankungen in den folgenden fünf Perioden auf etwas verminderter Höhe und sinkt in der letzten Periode auf das gleiche geringe Maß von Energie, welches die Zuwachsgröße bei Beginn der Greweihentwicklung gezeigt hatte.

Dieses abweichende Verhalten des Geweihwachstumsganges vom Rehbock im Vergleich zu dem des Edelhirsches durfte vielleicht eher als ein typisches, denn als ein zufälliges und auf individuelle Disposition zurickzufiuhrendes anzusehen sein. Indes müssen weitere und in gleichem Sinne wie die obigen auszuführende und anzustellende Untersuchungen und Beobachtnngen iiber das hier dargelegte Problem erst die Entscheidung bringen. 
Archie fir Entwicklungsmechanil. Bd XXV.

Tat. XVII.

Fig. 1.

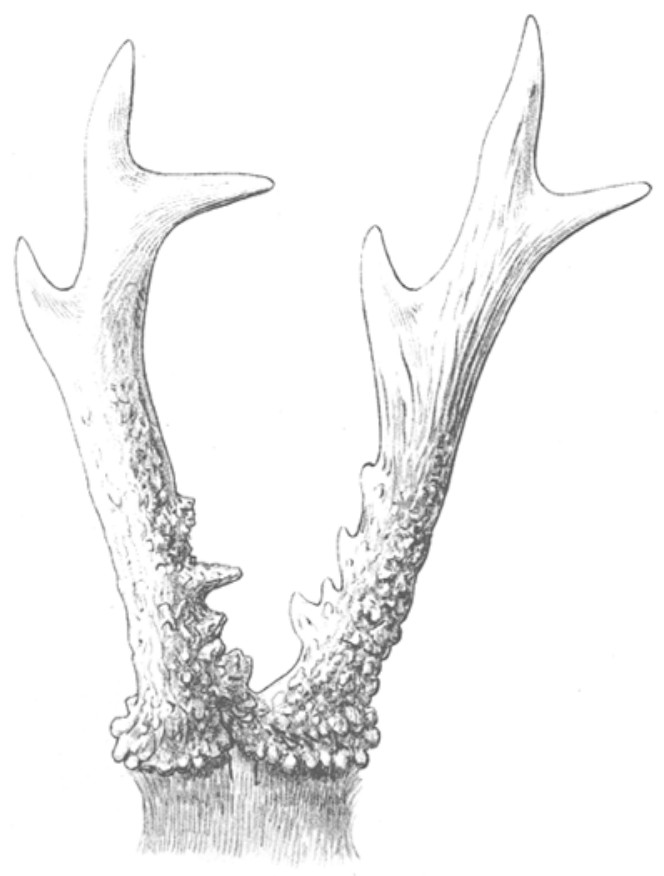

Fig. 2.

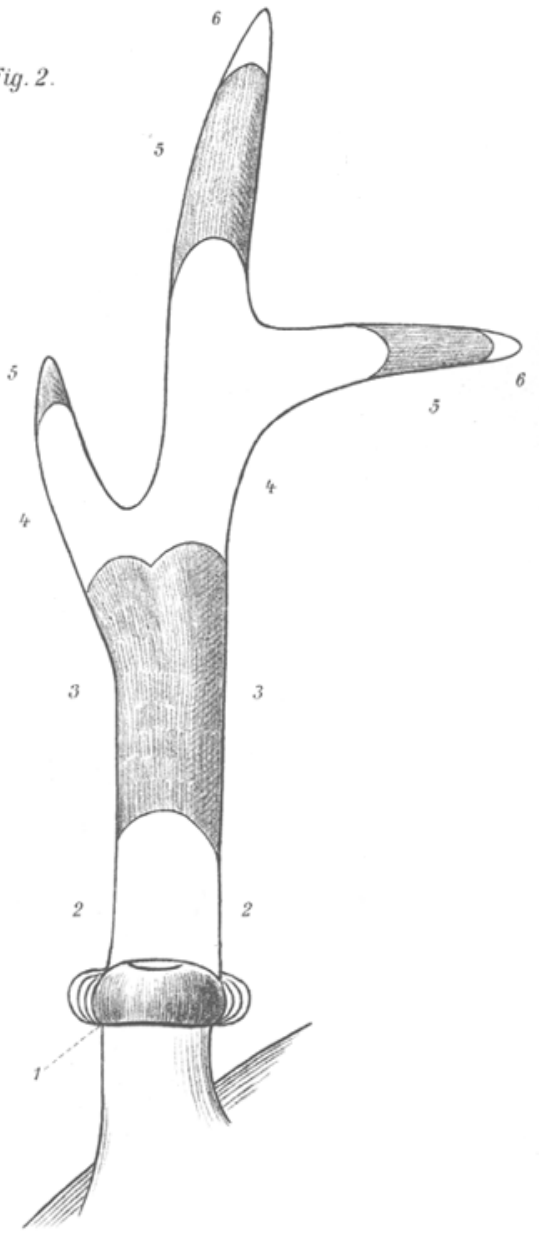

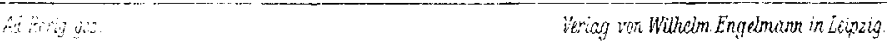

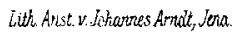

